\title{
ON MAXIMAL FUNCTIONS ASSOCIATED TO HYPERSURFACES AND THE CAUCHY PROBLEM FOR STRICTLY HYPERBOLIC OPERATORS
}

\author{
CHRISTOPHER D. SOGGE
}

\begin{abstract}
In this paper we prove a maximal Fourier integral theorem for the types of operators which arise in the study of maximal functions associated to averaging over hypersurfaces and also the Cauchy problem for hyperbolic operators. We apply the Fourier integral theorem to generalize Stein's spherical maximal theorem (see [8]) and also to prove a sharp theorem for the almost everywhere convergence to $L^{p}$ initial data of solutions to the Cauchy problem for second order strictly hyperbolic operators. Our results improve those of Greenleaf [3] and Ruiz [6]. We also can prove almost everywhere convergence to $L^{2}$ initial data for operators of order $m \geq 3$.
\end{abstract}

Introduction. In 1976, E. M. Stein [8] proved that if

$$
\left(M_{t} f\right)(x)=\int_{S^{n-1}} f(x+t y) d \sigma(y)
$$

then $(\mathcal{M} f)(x)=\sup _{t>0}\left|\left(M_{t} f\right)(x)\right|$ is bounded on $L^{p}\left(\mathbf{R}^{n}\right)$ when $p>n /(n-1)$ and $n \geq 3$. Also by considering a slight variant of this maximal theorem he was able to establish that if $L=(\partial / \partial t)^{2}-\sum_{j=1}^{n}\left(\partial / \partial x_{j}\right)^{2}$ then (weak) solutions to the Cauchy problem for $\mathbf{R}_{+}^{n+1}$,

$$
\left\{\begin{array}{l}
L u(t, x)=0, \\
u(0, x)=0, \\
(\partial u / \partial t)(0, x)=f(x),
\end{array}\right.
$$

have the property that $\lim _{t \rightarrow 0} u(t, x) / t=f(x)$ almost everywhere, whenever $f \in$ $L_{\text {loc }}^{p}\left(\mathbf{R}^{n}\right), n \geq 1,2 n /(n+1)<p$.

All of these results were obtained from interpolation with a certain key $L^{2}$ estimate. The latter was subsequently generalized by Sogge and Stein [7]. They showed that if a multiplier $m(\xi)$ is $C^{1}$ and satisfies the estimates $|m(\xi)|,|\nabla m(\xi)| \leq$ $C(1+|\xi|)^{-\delta}$ for some $\delta>1 / 2$, and if one defines $T_{t}$ by $\left(T_{t} f\right)^{-}(\xi)=m(t \xi) \hat{f}(\xi)$ (" " denotes the Fourier transform) then one has

$$
\left\|\sup _{t>0}\left|T_{t} f\right|\right\|_{L^{2}\left(\mathbf{R}^{n}\right)} \leq C\|f\|_{L^{2}\left(\mathbf{R}^{n}\right)} .
$$

The goal of this paper is to generalize all of these results to variable coefficient cases. Instead of dealing with multiplier operators we shall be considering Fourier

Received by the editors November 8, 1985 .

1980 Mathematics Subject Classification (1985 Revision). Primary 42B25, 35L15.

Supported in part by an NSF postdoctoral fellowship. 
integral operators of the type

$$
\left(T_{t} f\right)(x)=\int_{\mathbf{R}^{n}} \hat{f}(\xi) a(t ; x, t \xi) e^{i[\langle x, \xi\rangle+t \phi(t ; x, \xi)]} d \xi .
$$

Under certain regularity conditions on $a$ and $\phi$ we will show that if the amplitude satisfies certain decay conditions (roughly that $a(t ; x, \xi) \in S_{1,0}^{-\delta}$ uniformly in $t$ for $\delta>1 / 2$ ), then the following inequality holds for these operators:

$$
\left\|\sup _{0<t<1}\left|\left(T_{t} f\right)(x)\right|\right\|_{L^{2}\left(\mathbf{R}^{n}\right)} \leq C\|f\|_{L^{2}\left(\mathbf{R}^{n}\right)} .
$$

As above this will be the key estimate which we shall apply along with analytic interpolation to obtain other results.

The first result will be to generalize Stein's maximal theorem. Roughly speaking, we shall be assuming that $\left\{S_{t, x}\right\}, t \in \overline{\mathbf{R}}_{+}, x \in \mathbf{R}^{n}$, are smooth perturbations of a smooth hypersurface $S \subset \mathbf{R}^{n}$. We shall also require that the Gaussian curvature is nowhere vanishing on each $S_{t, x}$. Then if $\psi(x, y) \in C_{0}^{\infty}\left(\mathbf{R}^{n} \times \mathbf{R}^{n}\right)$ has the property that $\psi(x, y) d \sigma_{t, x}(y)$ is a compactly supported measure on $S_{t, x}\left(d \sigma_{t, x}\right.$ denotes Lebesgue measure on $S_{t, x}$ ), we show that there is a $t_{0}>0$ so that if for $t>0$ we now put

$$
\left(M_{t} f\right)(x)=\int_{S_{t, x}} f(x+t y) \psi(x, y) d \sigma_{t, x}(y)
$$

and $(\mathcal{M} f)(x)=\sup _{0<t<t_{0}}\left|\left(M_{t} f\right)(x)\right|$, then

$$
\|\mathcal{M} f\|_{L^{p}\left(\mathbf{R}^{n}\right)} \leq C\|f\|_{L^{p}\left(\mathbf{R}^{n}\right)}
$$

for the same range of $p$ and $n$ as Stein's spherical maximal function. Our maximal theorem is proved by noticing that, for small enough $t, M_{t} f$ is (up to a trivial error) a finite sum of Fourier integral operators $T_{t} f$ as above.

Variable coefficient maximal theorems were previously proved by A. Greenleaf [3] for hypersurfaces $S_{t, x}$ which in addition to the above conditions are also assumed to be compact, convex, and starlike with respect to the origin. Also, E. M. Stein and the author have proved more general results where one only assumes that the curvature does not vanish of infinite order (cf. Sogge and Stein [7]). The proof of the results in the present paper is simpler though and is of independent interest.

Our final set of applications to the maximal Fourier integral theorem concerns the pointwise convergence properties of solutions to Cauchy problems for strictly hyperbolic linear differential operators of order $m \geq 2$. More specifically, we shall deal with operators of the form

$$
L=\frac{\partial^{m}}{\partial t^{m}}-\sum_{j=0}^{m-1} A_{m-j}\left(t, x, D_{x}\right) \frac{\partial^{j}}{\partial t^{j}}
$$

$A_{m-j}$ being a differential operator of order $m-j$ with smooth coefficients. After placing a mild condition on $L$ to ensure that it has a finite domain of dependence (see Definition 3.2) we shall study the Cauchy problem

$$
\begin{cases}L u(t, x)=0, & t>0 \\ (\partial / \partial t)^{j} u(0, x)=0, & 0 \leq j<m-1 \\ (\partial / \partial t)^{m-1} u(0, x)=f(x) . & \end{cases}
$$


Given a smooth family of nontangential curves $\gamma(s, x)$ in $\mathbf{R}_{+}^{n+1}$ with $\gamma(0, x)=(0, x)$ our result will be that the (weak) solution to this Cauchy problem with initial data $f(x) \in L_{\text {loc }}^{p}\left(\mathbf{R}^{n}\right)$ has the property that

$$
u(\gamma(s, x)) / s^{m-1} \rightarrow c_{\gamma}(x) f(x),
$$

whenever $p>2 n /(n+2)$ for $m=2$ and $p=2$ for $m \geq 3$. Here $c_{\gamma}(x)$ is a smooth function which depends only on $\gamma(s, x)$. We remark that our methods can show that the result for $m \geq 3$ also holds for $f \in L^{2-\varepsilon_{m}}\left(\mathbf{R}^{n}\right)$ (in fact $\varepsilon_{m}=1$ for $m>n+1$ ), but since we cannot find the sharp $\varepsilon_{m}$ in general, we do not carry this out.

Results of this type were established by Greenleaf [3] and Ruiz [6] when one makes the additional assumption that the approach curves $\gamma(x, s)$ are time-like for each fixed $x$. Our result for $m \geq 3$ is new.

Our paper is organized as follows. In $\S 1$ we prove the maximal Fourier integral theorem while $\S 2$ concerns variable coefficient maximal theorems. $\S \S 3$ and 4 will be devoted to the Cauchy problem for operators of orders $m=2$ and $m \geq 3$ respectively. Also by $C$ we mean a constant which is not necessarily the same at each occurrence.

1. An $L^{2}$-maximal theorem. In this section we prove a maximal theorem for certain families of Fourier integral operators whose amplitudes satisfy certain decay conditions. More precisely, let $a(t ; x, \xi)$ and $\phi(t ; x, \xi)$ be smooth on $\overline{\mathbf{R}}_{+} \times \mathbf{R}^{n} \backslash 0$ and set

$$
\left(T_{t} f\right)(x)=\int_{\mathbf{R}^{n}} \hat{f}(\xi) a(t ; x, t \xi) e^{i[\langle x, \xi\rangle+t \phi(t ; x, \xi)]} d \xi .
$$
has

We are assuming that $a(t ; x, \xi)=0$ when $|\xi| \leq 2$ and also for some $\delta>1 / 2$ one

$$
\left|(\partial / \partial t)^{j}\left(D_{x}^{\alpha}\right)\left(D_{\xi}^{\beta}\right)\left(D_{\xi}^{\beta}\right) a(t ; x, \xi)\right| \leq C|\xi|^{-\delta-|\beta|}, \quad j \leq 1,|\alpha|,|\beta| \leq n+3 .
$$

Further, the phase functions $\phi$ are real, homogeneous of degree 1 in $\xi$ and satisfy

$$
\left|(\partial / \partial t)^{j}\left(D_{x}^{\alpha}\right)\left(D_{\xi}^{\beta}\right) \phi(t ; x, \xi)\right| \leq 1 / 2
$$

if $|\xi|=1, j \leq 1$, and $|\alpha|,|\beta| \leq n+3$. Then our result is the following.

THEOREM 1.1. Let $n \geq 1$. Then under the above hypotheses one has

$$
\left\|\sup _{0<t<1}\left|\left(T_{t} f\right)(x)\right|\right\|_{L^{2}\left(\mathbf{R}^{n}\right)} \leq C\|f\|_{L^{2}\left(\mathbf{R}^{n}\right)} .
$$

REMARK. Inequality (1.4) does not in general hold for operators whose amplitudes only satisfy (1.2) for $\delta=1 / 2$ (see [9]).

The proof of this inequality will require the following proposition, which at times will serve as a substitute for Plancherel's theorem for the Fourier transform. Results of this type are well known (see e.g. Treves [12]); however, for the sake of completeness we shall sketch the proof at the end of this section.

Proposition 1.1. Suppose $b(x, \xi), \psi(x, \xi)$ are $C^{(n+3)}\left(\mathbf{R}^{n} \times \mathbf{R}^{n} \backslash 0\right)$ with $b(x, \xi)=0$ when $|\xi| \leq 1$. Also suppose that $\left|D_{x}^{\alpha} D_{\xi}^{\beta} b(x, \xi)\right| \leq C_{0}|\xi|^{-|\beta|}$ if $|\alpha|,|\beta| \leq$ 
$n+3$, and that $\psi$ is real, homogeneous of degree 1 in $\xi$ and satisfies $\left|D_{x}^{\alpha} D_{\xi}^{\beta} \psi(x, \xi)\right| \leq$ $1 / 2$ if $|\xi|=1$ and $|\alpha|,|\beta| \leq n+3$. Set

$$
\mathcal{F}(g)(x)=\int_{\mathbf{R}^{n}} e^{i[\langle x, \xi\rangle+\psi(x, \xi)]} b(x, \xi) \hat{g}(\xi) d \xi .
$$

Then

$$
\|\mathcal{F}(g)\|_{2} \leq C\|g\|_{2}
$$

where $C$ depends only on $C_{0}$.

PROOF OF THEOREM 1.1. The proof of (1.4) involves taking fractional derivatives of the operators $T_{t}$ and then using square functions (cf. Sogge and Stein [7]).

Furthermore, since the argument for the most general case is not harder than the one for the case where the amplitudes and the phase functions are independent of $t$, we assume that this is the case to simplify the notation. Thus we shall deal with operators of the form

$$
\left(T_{t} f\right)(x)=\int \hat{f}(\xi) a(x, t \xi) e^{i[\langle x, \xi\rangle+t \phi(x, \xi)]} d \xi
$$

and we wish to show that (1.4) holds.

Now we recall the definition of a fractional derivative. Let $0<\alpha<1$; then for, say, bounded $F$ we define the fractional derivative of order $\alpha$ by

$$
I_{\alpha}(F)(t)=(\Gamma(\alpha))^{-1} \int_{0}^{t}(t-s)^{\alpha-1} F(s) d s .
$$

Then one easily checks that

$$
I_{\alpha}\left(I_{1-\alpha}(F)\right)=\int_{0}^{t} F(s) d s
$$

and so $I_{\alpha}\left(I_{1-\alpha}\left(F^{\prime}\right)\right)=F$ if $F(0)=0$.

With this in mind set

$$
T_{t}^{\alpha} f=(\Gamma(1-\alpha))^{-1} \int_{0}^{t}(t-s)^{-\alpha}(d / d s)\left(s^{\alpha} T_{s} f\right) d s
$$

Then one has $t^{\alpha} T_{t} f=I_{\alpha}\left(T_{t}^{\alpha} f\right)$. Consequently, by Schwarz's inequality if $1 / 2<$ $\alpha<1$ then

$$
\left|\left(T_{t}^{\alpha} f\right)(x)\right| \leq C_{\alpha}\left(t^{-1} \int_{0}^{t}\left|\left(T_{s}^{\alpha} f\right)(x)\right|^{2} d s\right)^{1 / 2} .
$$

In what follows we fix $\alpha<1$ satisfying $1 / 2<\alpha<\delta$. Then by (1.6), to prove (1.4) it is enough to show that

$$
\left\|\left(\int_{0}^{1}\left|\left(T_{s}^{\alpha} f\right)\right|^{2} d s / s\right)^{1 / 2}\right\|_{2} \leq C\|f\|_{2} .
$$

To verify this put

$$
\tilde{T}_{t}^{\alpha} f=(\Gamma(1-\alpha))^{-1} \int_{t / 2}^{t}(t-s)^{-\alpha}(d / d s)\left(s^{\alpha} T_{s} f\right) d s
$$


and set

$$
T_{t}^{\alpha} f=\tilde{T}_{t}^{\alpha}+\tilde{T}_{t}^{\alpha} f
$$

To handle this trivial term $\widetilde{T}_{t}^{\alpha}$ we notice from integration by parts that

$$
\begin{aligned}
\left(\int_{\mathbf{R}^{n}}\right. & \left.\int_{0}^{1}\left|\tilde{T}_{t}^{\alpha} f\right|^{2} d s / s d x\right)^{1 / 2} \\
\leq & C_{\alpha}\left(\int_{\mathbf{R}^{n}} \int_{0}^{1}\left|T_{s} f\right|^{2} d s / s d x\right)^{1 / 2} \\
& +C_{\alpha}\left(\int_{\mathbf{R}^{n}} \int_{0}^{1}\left|\int_{0}^{s / 2}(s-r)^{-\alpha-1} r^{\alpha} T_{r} f d r\right|^{2} d s / s d x\right)^{1 / 2} .
\end{aligned}
$$

The first of these last two expressions is easy to handle. In fact since $a(x, \xi)=0$ for $|\xi| \leq 1$, Proposition 1.1 and Plancherel's theorem imply that it is

$$
\leq C\left(\left.\left.\int_{0}^{1} \int_{|\xi|>1 / s}|\hat{f}(\xi)| s \xi\right|^{-\delta}\right|^{2} d \xi d s / s\right)^{1 / 2}=C^{\prime}\|f\|_{2} .
$$

Similarly, if one first applies a change of scale and Minkowski's integral inequality, then one sees that the second term is

$$
\leq \int_{0}^{1 / 2}(1-r)^{-\alpha-1} r^{\alpha}\left(\int_{\mathbf{R}^{n}} \int_{0}^{1}\left|T_{r s} f\right|^{2} d s / s d x\right)^{1 / 2} d r \leq C\|f\|_{2} .
$$

Consequently, we are left with estimating the nontrivial term:

$$
\begin{aligned}
& \left\|\left(\int_{0}^{1}\left|\tilde{T}_{s}^{\alpha} f\right|^{2} d s / s\right)^{1 / 2}\right\|_{2} \\
& \quad \cong\left(\int_{\mathbf{R}^{n}} \int_{0}^{1}\left|\int_{s / 2}^{s}(s-r)^{-\alpha}(d / d r)\left(r^{\alpha} T_{r} f\right) d r\right|^{2} d s / s d x\right)^{1 / 2} .
\end{aligned}
$$

To do so we use the following lemma.

LEMMA 1.1. Fix $\varepsilon$ satisfying $0<\varepsilon<\delta-\alpha$. Then for any $0<s \leq 1$ one has the inequality

$$
\left\|\int_{s / 2}^{s}(s-r)^{-\alpha}(d / d r)\left(r^{\alpha} T_{r} f\right) d r\right\|_{2} \leq C\left(\left.\left.\int_{|\xi|>1 / s}|\hat{f}(\xi)| s \xi\right|^{-\varepsilon}\right|^{2} d \xi\right)^{1 / 2} .
$$

If we use this estimate and (1.9) then once again Plancherel's theorem gives

$$
\left\|\left(\int_{0}^{1}\left|\tilde{T}_{s}^{\alpha} f\right|^{2} d s / s\right)^{1 / 2}\right\|_{2} \leq C\left(\left.\left.\int_{0}^{1} \int_{|\xi|>1 / s}|\hat{f}(\xi)| s \xi\right|^{-\varepsilon}\right|^{2} d \xi d s / s\right)^{1 / 2} \leq C^{\prime}\|f\|_{2} .
$$

Therefore, to finish our proof of Theorem 1.1 we need only prove Lemma 1.1. However, by a change of scale argument one easily sees that one can assume that 
$s=1$. Thus we wish to show that

$$
\left\|\int_{1 / 2}^{1}(1-r)^{-\alpha}(d / d r)\left(r^{\alpha} T_{r} f\right) d r\right\|_{2} \leq C\left(\left.\left.\int_{|\xi| \geq 1}|\hat{f}(\xi)| \xi\right|^{-\varepsilon}\right|^{2} d \xi\right)^{1 / 2} .
$$

But by integration by parts, the function inside the first $L^{2}$-norm equals

$$
\begin{aligned}
-\alpha \int_{\mathbf{R}^{n}} & \int_{1 / 2}^{1-|\xi|^{-1}}(1-r)^{-\alpha-1} r^{\alpha} \hat{f}(\xi) a(x, r \xi) e^{i[\langle x, \xi\rangle+r \phi(x, \xi)]} d r d \xi \\
& +\int_{|\xi|>2}|\xi|^{\alpha}\left(1-|\xi|^{-1}\right)^{\alpha} e^{i\left[\langle x, \xi\rangle+\left(1-|\xi|^{-1}\right) \phi(x, \xi)\right]} \\
& \times a\left(x,\left(1-|\xi|^{-1}\right) \xi\right) \hat{f}(\xi) d \xi-c\left(T_{1 / 2} f\right)(x) \\
& +\int_{\mathbf{R}^{n}} \int_{1-|\xi|^{-1}}^{1}(1-r)^{-\alpha}(d / d r)\left(r^{\alpha} e^{i[\langle x, \xi\rangle+r \phi(x, \xi)]} a(x, r \xi)\right) \hat{f}(\xi) d r d \xi \\
= & \mathrm{I}+\mathrm{II}+\mathrm{III} .
\end{aligned}
$$

We first estimate I. By changing the order of the integration and applying Minkowski's integral inequality one gets

$$
\begin{aligned}
\|\mathrm{I}\|_{2} & \cong\left(\int_{\mathbf{R}^{n}}\left|\int_{1 / 2}^{1} r^{\alpha}(1-r)^{-\alpha-1} \int_{|\xi|>(1-r)^{-1}} \hat{f}(\xi) a(x, r \xi) e^{i\langle\langle x, \xi\rangle+r \phi(x, \xi)]} d \xi d r\right|^{2} d x\right)^{1 / 2} \\
& \leq \int_{1 / 2}^{1} r^{\alpha}(1-r)^{-\alpha-1}\left(\int\left|\int_{|\xi|>(1-r)^{-1}} \hat{f}(\xi) a(x, r \xi) e^{i[\langle x, \xi\rangle+r \phi(x, \xi)]} d \xi\right|^{2} d x\right)^{1 / 2} d r .
\end{aligned}
$$

But by Proposition 1.1 this last expression is

$$
\begin{aligned}
& \leq C \int_{1 / 2}^{1} r^{\alpha}(1-r)^{-\alpha-1}\left(\left.\left.\int_{|\xi|>(1-r)^{-1}}|\hat{f}(\xi)| \xi\right|^{-\delta}\right|^{2} d \xi\right)^{1 / 2} d r \\
& \leq C\left(\left.\left.\int_{|\xi|>1}|\hat{f}(\xi)| \xi\right|^{-\varepsilon}\right|^{2} d \xi\right)^{1 / 2} \int_{1 / 2}^{1}(1-r)^{-\alpha-1+\delta-\varepsilon} d r \\
& =C^{\prime}\left(\left.\left.\int_{|\xi|>1}|\hat{f}(\xi)| \xi\right|^{-\varepsilon}\right|^{2} d \xi\right)^{1 / 2},
\end{aligned}
$$

as desired. The last equality comes from the fact that $\varepsilon<\delta-\alpha$.

One also sees from Proposition 1.1 that $\|\mathrm{II}\|_{2}$ also satisfies these bounds.

We now turn to III. To deal with this we first notice that

$$
(d / d r) e^{i r \phi(x, \xi)} a(x, r \xi)=\sum_{j=1}^{n} \xi_{j} e^{i r \phi(x, \xi)} a_{j}(x, r \xi)
$$

where the $a_{j}$ also satisfy (1.2). Therefore if, as before, we change the order of integration and then apply Minkowski's integral inequality and Proposition 1.1 
we get

$$
\begin{aligned}
\|\mathrm{III}\|_{2} & \leq \int_{1 / 2}^{1}(1-r)^{-\alpha} r^{\alpha}\left(\int_{\mathbf{R}^{n}} \mid \int_{1<|\xi|<(1-r)^{-1}} \hat{f}(\xi)(d / d r)\right. \\
& \left.\left.\times r^{\alpha} e^{i[\langle x, \xi\rangle+r \phi(x, \xi)]} a(x, r \xi)\right)\left.d \xi\right|^{2} d x\right)^{1 / 2} d r \\
& \leq C \int_{1 / 2}^{1} r^{\alpha}(1-r)^{-\alpha}\left(\left.\left.\int_{1<|\xi|<(1-r)^{-1}}|\hat{f}(\xi)| \xi\right|^{1-\delta}\right|^{2} d \xi\right)^{1 / 2} d r \\
& \leq C\left(\left.\left.\int_{|\xi|>1}|\hat{f}(\xi)| \xi\right|^{-\varepsilon}\right|^{2} d \xi\right)^{1 / 2} \int_{1 / 2}^{1}(1-r)^{-\alpha-1+\delta-\varepsilon} d r \\
& =C^{\prime}\left(\left.\left.\int_{|\xi|>1}|\hat{f}(\xi)| \xi\right|^{-\varepsilon}\right|^{2} d \xi\right)^{1 / 2} \cdot \quad \text { Q.E.D. }
\end{aligned}
$$

To finish this section we now sketch a proof of Proposition 1.1. We recall that we need to show $\|\mathcal{F}(g)\|_{2} \leq C\|g\|_{2}$, where

$$
\mathcal{F}(g)(x)=\int e^{i[\langle x, \xi\rangle+\psi(x, \xi)]} b(x, \xi) \hat{g}(\xi) d \xi,
$$

and $b$ and $\psi$ are as before.

Let $B$ denote the unit ball in $\mathbf{R}^{n}$. Then clearly the desired inequality will follow from the following:

$$
\int_{B}|\mathcal{F}(g)|^{2} d x \leq C \int|g(x)|^{2}(1+|x|)^{n-1} d x .
$$

However, after multiplying by a cutoff function, we see that we can now assume that the amplitude $b$ has $x$ support in the double of $B, B^{*}$.

Now let $g_{1}(x)=g(x)$ if $|x| \leq 3$ and $g_{1}(x)=0$ otherwise, and put $g=g_{1}+g_{2}$.

Then if $|x| \leq 1$ one easily sees from integration by parts that

$$
\left|\mathcal{F}\left(g_{2}\right)(x)\right| \leq C \int|g(y)|(1+|y|)^{-n-1} d y .
$$

By Schwarz's inequality this establishes (1.11) for $g_{2}$.

To finish the proof we notice that

$$
\int\left|\mathcal{F}\left(g_{1}\right)\right|^{2} d x \leq \iint\left|\hat{g}_{1}(\xi) \hat{g}_{1}(\eta)\right||K(\xi, \eta)| d \xi d \eta
$$

where

$$
K(\xi, \eta)=\int e^{i[\langle x, \xi-\eta\rangle+\psi(x, \xi)-\psi(x, \eta)]} b(x, \xi) \overline{b(x, \eta)} d x .
$$

Since $b$ has $x$-support in $B^{*}$ one sees from integrating by parts that

$$
|K(\xi, \eta)| \leq C(1+|\xi-\eta|)^{-n-1} .
$$

Therefore by the Schwarz inequality one gets the desired inequality for $g_{1}$.

This finishes the proof. 
2. Variable coefficient maximal theorems. In this section we prove a variable coefficient maximal theorem for hypersurfaces with nonvanishing Gaussian curvature. These results will follow from well-known stationary phase estimates along with our maximal Fourier integral theorem.

In our theorem we assume for each $t \in[0,1], x \in \mathbf{R}^{n}$ that $S_{t, x} \subset \mathbf{R}^{n}$ is a hypersurface given by $S_{t, x}=\left\{y \in \mathbf{R}^{n}: y \in \mathcal{O}, \Phi(t, x ; y)=0\right\}$, where 0 is an open set and the defining functions $\Phi$ are smooth on $[0,1] \times \mathbf{R}^{n} \times \mathbf{R}^{n}$ with $\nabla_{y} \Phi \neq 0$. Our main result of this section then is the following

THEOREM 2.1. Let $n \geq 3$ and let $S_{t, x}$ be as above. Suppose further that the Gaussian curvature is nowhere vanishing on each of the hypersurfaces and that $\psi \in C_{0}^{\infty}\left(\mathbf{R}^{n} \times 0\right)$. Put

$$
\left(M_{t} f\right)(x)=\int_{S_{t, x}} f(x+t y) \psi(x, y) d \sigma_{t, x}(y) .
$$

Then there is a $t_{0}>0$ so that if

$$
(\mathcal{M} f)(x)=\sup _{0<t \leq t_{0}}\left|\left(M_{t} f\right)(x)\right|,
$$

then for each $p, n /(n-1)<p \leq \infty$, one has

$$
\|\mathcal{M} f\|_{L^{p}\left(\mathbf{R}^{n}\right)} \leq C_{p}\|f\|_{L^{p}\left(\mathbf{R}^{n}\right)} .
$$

REMARKS. We must exercise some care in the precise definition of $\mathcal{M} f$. First one proves (2.1) for, say, $f \in C_{0}^{\infty}$, so that for such $f, f$ can be defined unambiguously. Next by the argument in $\left[\mathbf{9}\right.$, pp. 1285-1287] one can redefine $\left(M_{t} f\right)(x)$ for each $f \in L^{p}\left(\mathbf{R}^{n}\right)$ and every $0<t \leq t_{0}$ (on a set of $x$-measure zero) so that $\left(M_{t} f\right)(x)$ is continuous in $t$ for almost every $x$. Then $(2.1)$ holds for the resulting $(\mathcal{M} f)(x)=$ $\left.\sup _{0<t<t_{0}} \mid M_{t} f\right)(x) \mid$. Similar care has to be taken with the other variants of $(2.1)$ arising below.

It turns out to be more convenient to prove a local version of Theorem 2.1 which is in fact equivalent to it since $\psi(x, y)$ is compactly supported. In doing so we use the notation that if $x \in \mathbf{R}^{n}$ then $x=\left(x^{\prime}, x_{n}\right)$ where in what follows the "prime" always denotes a vector in $\mathbf{R}^{n-1}$. Also, by $B(y, \delta)$ we mean the ball of radius $\delta$ centered at $y$.

To formulate the local version we will now with an abuse of notation let $\psi\left(y^{\prime}\right) \in$ $C_{0}^{\infty}\left(\mathbf{R}^{n-1}\right)$. Also $h\left(t, x ; y^{\prime}\right)$ will be a smooth function on $[0,1] \times \mathbf{R}^{n} \times \mathbf{R}^{n-1}$ satisfying

$$
\left|\operatorname{det}\left(\partial^{2} h\left(t, x ; y^{\prime}\right) / \partial y_{j}^{\prime} \partial y_{k}^{\prime}\right)\right|>0 \quad \text { if } \psi\left(y^{\prime}\right) \neq 0 \text {. }
$$

We now set

$$
\left(M_{t}^{0} f\right)(x)=\int_{\mathbf{R}^{n-1}} f\left(x^{\prime}+t y^{\prime}, x_{n}+t h\left(t, x ; y^{\prime}\right)\right) \psi\left(y^{\prime}\right) d y^{\prime} .
$$

Then (2.1) will be equivalent to the statement that for such functions one can always find $\delta, t_{0}>0$ so that if $\left(\mathcal{M}^{0} f\right)(x)=\max _{0<t \leq t_{0}}\left|\left(M_{t}^{0} f\right)(x)\right|$ then

$$
\left(\int_{B(0, \delta)}\left|\left(\mathcal{M}^{0} f\right)(x)\right|^{p} d x\right)^{1 / p} \leq C_{p}\|f\|_{p}, \quad p>n /(n-1) .
$$


To prove $\left(2.1^{\prime}\right)$ we use analytic interpolation. For complex $\gamma$ and a fixed $\eta \in$ $C_{0}^{\infty}(\mathbf{R})$ satisfying $\eta(0)=1$, we consider

$$
\left(M_{t}^{\gamma} f\right)(x)=\frac{e^{\gamma^{2}}}{\Gamma(\gamma / 2)} \int_{\mathbf{R}^{n}} f(x+t y)\left|y_{n}-h(t, x ; y)\right|^{-1+\gamma} \psi\left(y^{\prime}\right) \eta\left(y_{n}-h\left(t, x, y^{\prime}\right)\right) d y
$$

for $f \in C_{0}^{\infty}\left(\mathbf{R}^{n}\right)$, where the integral is defined by analytic continuation in $\gamma$ for $\operatorname{Re}(\gamma) \leq 0$. In particular, when $\gamma=0$ the definition of $\left(M_{t}^{0} f\right)(x)$ in (2.2) agrees with that of (2.3). With this in mind we now state a result which contains $\left(2.1^{\prime}\right)$.

THEOREM 2.2. Let $n \geq 3$ and let $M_{t}^{\gamma}$ be as above. Then there are numbers $\delta, t_{0}>0$ so that if $\left(\mathcal{M}^{\gamma} f\right)(x)=\sup _{0<t \leq t_{0}}\left|\left(M_{0}^{\gamma} f\right)(x)\right|$ then the inequality

$$
\left\|\mathcal{M}^{\gamma} f\right\|_{L^{p}(B(0, \delta))} \leq C_{p, \gamma}\|f\|_{L^{p}\left(\mathbf{R}^{n}\right)}
$$

holds in the following circumstances:

(a) If $1<p \leq 2$ when $\operatorname{Re}(\gamma)>1-n+n / p$.

(b) If $2 \leq p \leq \infty$ when $\operatorname{Re}(\gamma)>(2-n) / p$.

To apply analytic interpolation we first notice that one plainly has the inequality

$$
\left\|\mathcal{M}^{\gamma} f\right\|_{L^{\infty}(B(0,1))} \leq C_{\varepsilon}\|f\|_{L^{\infty}} \text { when } \operatorname{Re}(\gamma) \geq \varepsilon>0 .
$$

Also, the Hardy-Littlewood maximal theorem implies that for $p>1$,

$$
\left\|\mathcal{M}^{\gamma} f\right\|_{L^{p}(B(0,1))} \leq C_{p}\|f\|_{L^{p}} \quad \text { if } \operatorname{Re}(\gamma)=1 .
$$

From these two inequalites and an analytic interpolation argument one easily sees that Theorem 2.2 is a consequence of the following.

PROPOSITION 2.1. Let $n \geq 3$. Then there are numbers $\delta, t_{0}<0$ so that if $\mathcal{M}^{\gamma} f$ is as in Theorem 2.2 then if $\operatorname{Re}(\gamma)>1-n / 2$

$$
\left\|\mathcal{M}^{\gamma} f\right\|_{L^{2}(B(0, \delta))} \leq C\|f\|_{L^{2}\left(\mathbf{R}^{n}\right)} .
$$

Here $C$ is a constant which depends only on $\operatorname{Re}(\gamma)$.

As we shall shortly see, Proposition 2.1 follows from our maximal Fourier integral theorem. To make this clear we shall need the following two well-known results. The first is Morse's lemma with parameters (see [4, Vol. III, pp. 502-503]) while the second lemma is a standard result from stationary phase (see [4, Vol. I, pp. 216-219]).

LEMMA 2.1. Let $g(x, y)\left(x \in \mathbf{R}^{n_{1}}, y \in \mathbf{R}^{n_{2}}\right)$ be a real-valued smooth function in a neighborhood of $(0,0)$. Assume that $\left(\nabla_{x} f\right)(0,0)=0$ and that the matrix $A=$ $\left(\partial^{2} f(0,0) / \partial x_{j} \partial x_{k}\right)$ is nonsingular. Then the equation $\left(\nabla_{x} f\right)(x, y)=0$ determines in a neighborhood of 0 a $C^{\infty}$ function $x(y)$ with $x(0)=0$. Furthermore, we have in a neighborhood of $(0,0)$

$$
f(x, y)=f(x(y), y)+\langle A z, z\rangle
$$

where $z$ is a $C^{\infty}$ function of $(x, y)$ and the Jacobian matrix $(\partial z / \partial x)^{-1}$ has bounded $C^{(N)}$ norms for each $N$ and every $(x, y)$ in this neighborhood of $(0,0)$.

LEMMA 2.2. (a) Let $u \in c_{0}^{\infty}\left(\mathbf{R}^{n}\right)$ and $f \in C^{\infty}\left(\mathbf{R}^{n}\right)$. Then for $\lambda>0$ one has

$$
\lambda^{k}\left|\int u(x) e^{i \lambda f(x)} d x\right| \leq C \sum_{|\alpha| \leq k} \sup \left(\left|D^{\alpha} u\right||\nabla f|^{|\alpha|-2 k}\right)
$$


(b) Let $A$ be a real symmetric nondegenerate matrix. Then we have for every integer $k>0$ and integer $s>n / 2$

$$
\begin{array}{r}
\left|\int u(x) e^{i \lambda\langle A x, x\rangle / 2} d x-(\operatorname{det}(\lambda A / 2 \pi i))^{-1 / 2} T_{k}(\lambda)\right| \\
\leq C j_{k}\left|\operatorname{det}\left(A^{-1}\right) / \lambda\right|^{n / 2+k} \sum_{|\alpha| \leq 2 k+s}\left\|D^{\alpha} u\right\|_{L^{2}},
\end{array}
$$

where

$$
T_{k}(\lambda)=\sum_{j=0}^{k-1}(2 i \lambda)^{-j}\left\langle A^{-1} D, D\right\rangle^{j} u(0) / j ! .
$$

We are now ready to prove Proposition 2.1. We recall that $\left(\partial^{2} h\left(t, x ; y^{\prime}\right) / \partial y_{j}^{\prime} \partial y_{k}^{\prime}\right)$ is nonsingular when $\psi\left(t^{\prime}\right) \neq 0$. Therefore we can apply Lemma 2.1 to the function

$$
\left\langle\omega,\left(y^{\prime}, h\left(t, x ; y^{\prime}\right)\right)\right\rangle, \quad \omega \in S^{n-1},
$$

in a neighborhood of $\omega=\omega_{0}, t=0, x=0, y^{\prime}=y_{0}^{\prime}$ if

$$
\psi\left(y_{0}^{\prime}\right) \neq 0 \quad \text { and } \quad \nabla_{y^{\prime}}\left(\left\langle\omega_{0},\left(y^{\prime}, h\left(t, x, ; y^{\prime}\right)\right)\right\rangle\right)=0 .
$$

Furthermore, since $\psi$ has compact support, one easily sees from Lemmas 2.1 and 2.2 that one can rewrite $\psi$ as a finite sum $\psi=\sum_{j=1}^{N} \psi_{j}$, where $\psi_{j} \in C_{0}^{\infty}\left(\mathbf{R}^{n-1}\right)$ and where the $\psi_{j}$ have small enough support that for $(t, x)$ near $(0,0)$ we can obtain a favorable expression for

$$
\Omega_{j}(t ; x, \xi)=\int_{\mathbf{R}^{n-1}} e^{2 \pi i\left\langle\xi,\left(y^{\prime}, h\left(t, x ; y^{\prime}\right)\right)\right\rangle} \psi_{j}\left(y^{\prime}\right) d y^{\prime} .
$$

More precisely, there must be a $\delta>0$ so that if $0 \leq t,|x| \leq \delta$ and if each $\psi_{j}$ is supported in a ball of radius $\delta$ then for $|\xi| \geq 1$

$$
\Omega_{j}(t ; x, \xi)=e^{i \phi_{j}(t ; x, \xi)} a_{j}(t ; x, \xi),
$$

where $\phi_{j}$ and $a_{j}$ are smooth on $\{(t, x, \xi): 0 \leq t,|x| \leq \delta,|\xi|>1\}, \phi_{j}$ is real and homogeneous of degree one in $\xi$ and $a_{j}$ satisfies the estimate

$$
\left|(\partial / \partial t)^{k}\left(D_{x}^{\alpha}\right)\left(D_{\xi}^{\beta}\right) a_{j}(t ; x, \xi)\right| \leq C|\xi|^{-(n-1) / 2-|\beta|},
$$

whenever $|\xi| \geq 1,0 \leq t,|x| \leq \delta, k \leq 1$, and $|\alpha|,|\beta| \leq n+3$.

To apply this we notice from (2.3) that

$$
\left(M_{t}^{\gamma} f\right)(x)=\sum_{j} \int \hat{f}(\xi) \Omega_{j}(t ; x, t \xi) \hat{\eta}_{\gamma}\left(t \xi_{n}\right) e^{-2 \pi i\langle x, \xi\rangle} d \xi
$$

where

It is well known that $\hat{\eta}_{\gamma}$ is $C^{\infty}$ and

$$
\eta_{\gamma}\left(y_{n}\right)=\left(e^{\gamma^{2}} / \Gamma(\gamma / 2)\right)\left|y_{n}\right|^{-1+\gamma} \eta\left(y_{n}\right) .
$$

$$
\left|\left(d / d \xi_{n}\right)^{k} \hat{\eta}_{\gamma}\left(\xi_{n}\right)\right| \leq C_{\gamma, k}\left(1+\left|\xi_{n}\right|\right)^{-\operatorname{Re}(\gamma)-k} .
$$

Consequently, if $\operatorname{Re}(\gamma)>1-n / 2$ and if $\chi \in C^{\infty}\left(\mathbf{R}^{n}\right)$ has the property that $\chi(\xi)=0$ if $|\xi| \leq 1$ and $\chi(\xi)=1$ if $|\xi| \geq 2$ then

$$
\left|(\partial / \partial t)^{k}\left(D_{x}^{\alpha}\right)\left(D_{\xi}^{\beta}\right) \chi(\xi) a_{j}(t ; x, \xi) \hat{\eta}_{\gamma}\left(\xi_{n}\right)\right| \leq C|\xi|^{-1 / 2-\varepsilon-|\beta|}
$$

for some $\varepsilon>0$ whenever $0 \leq t,|x| \leq \delta, k \leq 1$, and $|\alpha|,|\beta| \leq n+3$. 
From (2.8) and Theorem 1.1 one sees that if

$$
\left(T_{t}^{j} f\right)(x)=\int \hat{f}(\xi) \chi(t \xi) a_{j}(t ; x, t \xi) \hat{\eta}_{\gamma}\left(t \xi_{n}\right) e^{2 \pi i\left[t \phi_{j}(t ; x, \xi)-\langle x, \xi\rangle\right]} d \xi
$$

then there must be a $t_{0} \leq \delta$ so that

$$
\left\|\sup _{0<t \leq t_{0}}\left|\left(T_{t}^{j} f\right)(x)\right|\right\|_{L^{2}(B(0, \delta / 2))} \leq C\|f\|_{L^{2}\left(\mathbf{R}^{n}\right)} .
$$

Furthermore, since for $0<t \leq \delta, 0 \leq|x| \leq \delta$,

$$
\left|\int \hat{f}(\xi)(1-\chi(t \xi)) \Omega_{j}(t ; x, t \xi) \hat{\eta}_{\gamma}\left(t \xi_{n}\right) e^{-2 \pi i\langle x, \xi\rangle} d \xi\right| \leq C f^{*}(x)
$$

(where as before $f^{*}$ denotes the Hardy-Littlewood maximal function), one concludes from (2.7)-(2.10) that

$$
\left\|\sup _{0<t \leq t_{0}} \mid\left(M_{t}^{\gamma} f\right)(x)\right\|\left\|_{L^{2}(B(0, \delta / 2))} \leq C\right\| f \|_{L^{2}\left(\mathbf{R}^{n}\right)}
$$

whenever $\operatorname{Re}(\gamma)>1-n / 2$.

This finishes the proof of Proposition 2.1 which in turn finishes the proofs of Theorems 2.1 and 2.2 .

3. The Cauchy problem for strictly hyperbolic operators of order $m=2$. Before stating our results for operators of order two it will be convenient to review the properties of strictly hyperbolic operators which will be needed in the next two sections. We shall study the Cauchy problem for $\mathbf{R}_{+}^{n+1}$ :

$$
\left\{\begin{array}{l}
L u=0 \\
\frac{\partial^{j} u}{\partial t^{j}}(0, x)=0, \quad 0 \leq j<m-1 \\
\frac{\partial^{m-1} u}{\partial t^{m-1}}(0, x)=f(x)
\end{array}\right.
$$

for certain operators $L$ of the form

$$
L=\frac{\partial^{m}}{\partial t^{m}}-\sum_{j=0}^{m-1} A_{m-j}\left(t, x, \partial_{x}\right) \frac{\partial^{j}}{\partial t^{j}} .
$$

Here $A_{m-j}$ is a differential operator of order $m-j$ with smooth coefficients which, for simplicity, we assume to be constant off of a compact set. If $A_{m-j}$ has the top order symbol $\tilde{A}_{m-j}$ then the principal part of $L, L_{m}$, has the symbol

$$
L_{m}(t, x, \tau, \xi)=\tau^{m}-\sum_{j=0}^{m-1} \tilde{A}_{m-j}(t, x, \xi) \tau^{j}
$$

DEFINITION 3.1. $L$ is said to be strictly hyperbolic if for each $x_{0} \in \mathbf{R}^{n}, t_{0} \in \mathbf{R}$, and $\xi \in \mathbf{R}^{n} \backslash 0$ the roots of the polynomial in $\tau, L_{m}\left(t_{0}, x_{0}, \tau, \xi\right)$ are real and distinct.

EXAMPLES. The wave operator $\partial^{2} / \partial t^{2}-\sum_{j=1}^{n} \partial^{2} / \partial x_{j}^{2}$ is a second order strictly hyperbolic operator. Also, the operators of crystal optics in $\mathbf{R}^{3}$ whose symbol is given by $L(\tau, \xi)=\tau^{4}-\tau^{2} \Psi(\xi)+|\xi|^{2} \Phi(\xi)$, where $\Psi(\xi)=\sum_{j=1}^{3}\left(|\xi|^{2}-\xi_{j}^{2}\right) / \sigma_{j}$ and 
$\Phi(\xi)=\xi_{1}^{2} / \sigma_{2} \sigma_{3}+\xi_{2}^{2} / \sigma_{1} \sigma_{3}+\xi_{3}^{2} / \sigma_{1} \sigma_{2}$ for the appropriate constants $\sigma_{j}$ relating to the medium of the crystal in the axial directions, are examples of strictly hyperbolic operators of order four (see e.g. [2]).

Since we want to obtain a result for operators with variable coefficients it is convenient to recall the notion of the domain of dependence for solutions of (3.1). This allows us to place a natural condition on the coefficients which guarantee the existence of a favorable representation (for small enough $t>0$ ) of the solutions $u(t, x)$ of $(3.1)$.

DEFinition 3.2. Given $\left(t_{0}, x_{0}\right) \in \mathbf{R}_{+}^{n+1}$ we say that $\Omega \subset \mathbf{R}^{n}$ is a domain of dependence of $\left(t_{0}, x_{0}\right)$ for the Cauchy problem (3.1) if whenever $f \in C_{0}^{\infty}\left(\mathbf{R}^{n}\right)$ has the property that $f=0$ on $\Omega$ then $u\left(t_{0}, x_{0}\right)=0$.

Since the domain of dependence of $\left(t_{0}, x_{0}\right)$ is related to the "timelike directions" at $\left(t_{0}, x_{0}\right)$ we also recall the following definition.

DEFINITION 3.3. $V \in \mathbf{R}^{n+1}$ is said to be a timelike direction for the strictly hyperbolic operator $L$ at $\left(t_{0}, x_{0}\right)$ if the equation

$$
L_{m}\left(t_{0}, x_{0}, X+\tau V\right)=0
$$

has $m$ distinct roots $\tau_{1}, \ldots, \tau_{m}$ whenever $X$ is not proportional to $V$.

Next we note the following well-known result (see e.g. $[\mathbf{1 1}$, p. 81]).

LEMMA 3.1. Suppose that $L$ is strictly hyperbolic. Then if for every $\left(t_{0}, x_{0}\right) \in$ $\mathbf{R}_{+}^{n+1}$ the vector $\left(\nu_{0}, \nu\right) \in \mathbf{R}^{n+1}\left(\nu \in \mathbf{R}^{n}\right)$ is timelike whenever

$$
\frac{|\nu|}{\left|\nu_{0}\right|} \leq C_{0}^{-1}
$$

it then follows that $\left(t_{0}, x_{0}\right)$ has the bounded domain of dependence $B\left(x_{0}, C_{0} t_{0}\right)$.

The operators which we shall consider will all satisfy (3.2). This is a mild condition for the coefficients which is satisfied for instance if the coefficients of the operator $L$ are constant outside a compact set.

Since we want to study the convergence properties of solutions to (3.1) along curves we make the following definition.

DEFINITION 3.4. We say that $s \rightarrow \gamma(s, x)$ is a smooth family of nontangential curves if the following conditions are met:

(i) $\gamma$ is in $C^{\infty}\left(\mathbf{R}^{n+1}\right)$ and has bounded derivatives.

(ii) $\gamma(0, x)=(0, x)$ and $\gamma(s, x) \in \mathbf{R}_{+}^{n+1}$ if $s>0$.

(iii) If we write $\partial \gamma / \partial s=\left(\gamma_{0}^{\prime}, \gamma_{1}^{\prime}, \ldots, \gamma_{n}^{\prime}\right)$ then there is an absolute constant $C$ for which

$$
\left|\left(\gamma_{1}^{\prime}, \ldots, \gamma_{n}^{\prime}\right)\right| / \gamma_{0}^{\prime} \leq C .
$$

REMARK. It is necessary to place some sort of nontangency condition on the approach curves if one hopes to have $u(\gamma(s, x)) /\left(\gamma_{0}(s, x)\right)^{m-1}$ tend to a multiple of $f(x)$ a.e. $s \rightarrow 0$ for $f \in L^{p}$. (Here we have written $\gamma=\left(\gamma_{0}, \gamma_{1}, \ldots, \gamma_{n}\right)$.) In fact, if $L=\partial^{2} / \partial t^{2}-\partial^{2} / \partial x^{2}$ is the wave operator for $\mathbf{R}^{2}$ then solutions for (3.1) are given by

$$
u(t, x)=\int_{-t}^{t} f(x+y) d y
$$


and so a classical theorem of Littlewood implies that if $\gamma$ is a curve in $\mathbf{R}^{2}$ with $\gamma(0)=0$ and $\gamma_{0}^{\prime}(0)=0$ then there are $g \in L^{p}(\mathbf{R})(1<p<\infty)$ for which $u((0, x)+\gamma(s)) / \gamma_{0}(s)$ does not tend to a limit for almost every $x$.

Having dealt with these preliminaries we finally are ready to state the following well-known result for small time solutions of (3.1) (see e.g. [12, pp. 308-313] and [2, pp. 215-221]). This lemma will allow us to use our maximal Fourier integral Theorem 1.1.

LEMMA 3.2. Let the strictly hyperbolic operator of order $m \geq 1$ acting on $\mathbf{R}^{n+1}, n \geq 1$, be as above and assume that $L$ satisfies (3.2). Then there is a $t_{0}>0$ so that for $0<t \leq t_{0}$ the solution $u(t, x)$ of the Cauchy problem satisfies

$$
u(t, x) / t^{m-1}=\sum_{k=0}^{m-1} \int \hat{f}(\xi) a_{k}(t ; x, t \xi) e^{-2 \pi i\left[\langle x, \xi\rangle+t \phi_{k}(t ; x, \xi)\right]} d \xi+(R f)(t, x) .
$$

Here $\phi_{k}(t ; x, \xi)$ and $a_{k}(t ; x, \xi)$ are smooth on $\left(0, t_{0}\right] \times \mathbf{R}^{n} \times \mathbf{R}^{n} \backslash 0$ and $a_{k}(t ; x, \xi)=0$ if $|\xi| \leq 1$ and $a_{k}$ satisfies $(1.2)$ with $\delta=m-1$. Also $\phi_{k}(t ; x, \xi)$ is real, homogeneous of degree one in $\xi$ and satisfies

$$
\left|(\partial / \partial t)^{j}\left(D_{x}^{\alpha}\right)\left(D_{\xi}^{\beta}\right) \phi_{k}(t ; x, \xi)\right| \leq C
$$

if $|\xi|=1, j \leq 1$, and $|\alpha|,|\beta| \leq n+3$. Finally the remainder term satisfies the estimate

$$
|(R f)(t, x)| \leq C \int t^{-n}(1+|(x-y) / t|)^{-n-1}|f(y)| d y .
$$

We remark that one can only in general get the formula (3.4) for a small range of $t$ since "the phase function" $\langle x, \xi\rangle+t \phi(t ; x, \xi)$ satisfies a nonlinear differential equation-the so-called eikonal equation.

We now focus our attention on the case where $m=2$. We have finally developed the necessary background to state and prove the main result of this section.

THEOREM 3.1. Let $\gamma(s, x)=\left(\gamma_{0}(s, x), \ldots, \gamma_{n}(s, x)\right)$ be a smooth family of nontangential curves in $\mathbf{R}^{n+1}, n \geq 1$. Also let $L$ be a second order strictly hyperbolic operator as above which satisfies (3.2). Then if $f \in L_{\text {loc }}^{p}\left(\mathbf{R}^{n}\right), 2 n /(n+1)<p$, the (weak) solution $u(t, x)$ of the Cauchy problem (3.1) satisfies

$$
u(\gamma(s, x)) / \gamma_{0}(s, x) \rightarrow c_{\gamma}(x) f(x) \quad \text { as } s \rightarrow 0_{+}
$$

almost everywhere; the smooth function $c_{\gamma}(x)$ is given by

$$
c_{\gamma}(x)=\left\lfloor\left(\partial \gamma_{0} / \partial s\right)(0, x)\right\rfloor^{-1} \text {. }
$$

PROOF OF THEOREM 3.1. In order to prove (3.7) it is enough to show that there is an $s_{0}>0$ so that if $2 n /(n+1)<p \leq 2$ then

$$
\left\|\sup _{0<s \leq s_{0}}\left|u(\gamma(s, x)) / \gamma_{0}(s, x)\right|\right\|_{L^{p}\left(\mathbf{R}^{n}\right)} \leq C_{p}\|f\|_{L^{p}\left(\mathbf{R}^{n}\right)} .
$$

In fact since $L$ has bounded domain of dependence, (3.7) will follow from (3.8) by a standard limiting argument which uses the fact that (3.7) holds for (say) $f \in C_{0}^{\infty}\left(\mathbf{R}^{n}\right)$. 
To prove (3.8) we let $1 \leq k \leq 2$ and put

$$
\begin{aligned}
& \tilde{a}(s ; x, \xi)=a_{k}\left(\gamma_{0}(s, x) ; \tilde{\gamma}(s, x),\left(\gamma_{0}(s, x) / s\right) \xi\right), \\
& \tilde{\phi}(s ; x, \xi)=\left(\gamma_{0}(s, x) / s\right) \cdot \phi_{k}\left(\gamma_{0}(s, x) ; \tilde{\gamma}(s, x), \xi\right)-\langle(\tilde{\gamma}(s, x)-x) / s, \xi\rangle,
\end{aligned}
$$

where $\tilde{\gamma}(s, x)=\left(\gamma_{1}(s, x), \ldots, \gamma_{n}(s, x)\right)$. Also, set

$$
\left(M_{s} f\right)(x)=\int \hat{f}(\xi) e^{-2 \pi i[\langle x, \xi\rangle+s \tilde{\phi}(s ; x, \xi)]} \tilde{a}(s ; x, s \xi) d \xi .
$$

Then since $\tilde{\gamma}(s, x)=x+E(s, x)$, where $E$ is smooth and $|E(s, x)| \leq C s$ one sees from (3.4) and (3.6) that it is enough to show that there is an $s_{0}>0$ for which

$$
\left\|\sup _{0<s \leq s_{0}} \mid\left(M_{s} f\right)(x)\right\|\left\|_{L^{p}\left(\mathbf{R}^{n}\right)} \leq C_{p}\right\| f \|_{L^{p}\left(\mathbf{R}^{n}\right)}, \quad p>2 n /(n+1) .
$$

To prove this inequality we shall use interpolation. For this purpose define for complex $\gamma$

$$
\left(M_{s}^{\gamma} f\right)(x)=e^{\gamma^{2}} \int \hat{f}(\xi)|s \xi|^{\gamma} \tilde{a}(s ; x, s \xi) e^{-2 \pi i[\langle x, \xi\rangle+s \tilde{\phi}(s ; x, \xi)]} d \xi .
$$

Then it is clear that (3.9) follows via an analytic interpolation argument from the following two lemmas.

LEMMA 3.3. If $\operatorname{Re}(\gamma)<1 / 2$ there is a constant $C$ which depends only on $\operatorname{Re}(\gamma)$ such that for some $s_{0}>0$ one has

$$
\left\|\sup _{0<s \leq s_{0}}\left|\left(M_{s}^{\gamma} f\right)(x)\right|\right\|_{L^{2}\left(\mathbf{R}^{n}\right)} \leq C\|f\|_{L^{2}\left(\mathbf{R}^{n}\right)} .
$$

LEMMA 3.4. Let $\gamma$ satisfy $\operatorname{Re}(\gamma)<-(n-1) / 2$. Then there is an $s_{0}>0$ and a constant $C_{p}$ depending only on $p$ and $\operatorname{Re}(\gamma)$ for which

$$
\left\|\sup _{0<s \leq s_{0}}\left|\left(M_{s}^{\gamma} f\right)(x)\right|\right\|_{L^{p}\left(\mathbf{R}^{n}\right)} \leq C_{p}\|f\|_{L^{p}\left(\mathbf{R}^{n}\right)}, \quad 1<p \leq \infty .
$$

To verify Lemma 3.3 we recall that $\gamma(x, s)$ is a smooth family of nontangential curves. One easily sees that this implies that $\tilde{\phi}$ satisfies (3.5) and also implied that $e^{\gamma^{2}}|\xi|^{\gamma} a(s ; x, \xi)$ satisfies (1.2) for some $\delta>1 / 2$, and so Lemma 3.3 follows from Theorem 1.1 .

By the Hardy-Littlewood maximal theorem in order to prove Lemma 3.4, it suffices to show that for some $s_{0}>0$ there are constants $C$ as in Lemma 3.4 so that if $\operatorname{Re}(\gamma)<-(n-1) / 2$ and $0<s \leq s_{0}$ then

$$
\left.\left|\int e^{-2 \pi i[\langle(x-y), \xi\rangle+s \tilde{\phi}(s ; x, \xi)]} e^{\gamma^{2}}\right| s \xi\right|^{\gamma} \tilde{a}(s ; x, s \xi) d \xi \mid \leq C s^{-n}(1+|(x-y) / s|)^{-n-1} .
$$

When $n=1$ this inequality follows easily by integration by parts since in this case $|\xi|^{\gamma} a(s ; x, \xi)$ is in $L^{1}(\mathbf{R}, d \xi)$ uniformly. Therefore, we assume that $n \leq 2$ from now on.

But by (3.9)

$$
\langle x-y, \xi\rangle+s \tilde{\phi}(s ; x, \xi)=\langle(\tilde{\gamma}(s, x)-y), \xi\rangle+\gamma_{0}(s, x) \phi_{k}\left(\gamma_{0}(s, x) ; \tilde{\gamma}(s, x), \xi\right),
$$


and therefore since $\gamma$ is nontangential it is enough to prove the inequality where in (3.11) we replace $s \tilde{\phi}(s ; x, \xi)$ by $\gamma_{0}(s, x) \phi_{k}\left(\gamma_{0}(s, x) ; \tilde{\gamma}(s, x), \xi\right)$. The latter function is homogeneous of degree one in $\xi$. Moreover, since $\phi_{k}$ satisfies the eikonal equation (see e.g. [1, pp. 49-50]) it is well known that there is a $t_{0}<0$ so that the following two properties hold when $n \geq 2$.

(i) If $0<t \leq t_{0}$ then $\phi_{k}(t ; x, \xi)$ is either positive or negative for all $\xi \in \mathbf{R}^{n} \backslash 0$.

(ii) If $0<t \leq t_{0}$ then for every $(t, x)$ the hypersurface

$$
\left\{\xi: \phi_{k}(t ; x, \xi)= \pm 1\right\}
$$

is convex and has the property that its Gaussian curvature is always bounded below by some positive number.

Finally, the above remarks and a change of scale argument will show that (3.11) is a consequence of the following lemma.

LEMMA 3.5. Suppose that $\Phi(\xi) \in C^{\infty}\left(\mathbf{R}^{n} \backslash 0\right), n \geq 2$, is homogeneous of degree one, positive on $\mathbf{R}^{n} \backslash 0$ and that the surface $S=\{\xi: \Phi(\xi)=1\}$ is convex with Gaussian curvature bounded below by $c_{0}>0$. Also assume that $b(\xi) \in C^{\infty}\left(\mathbf{R}^{n}\right)$ has the properties that $b(\xi)=0$ for $|\xi| \leq 1$ and

$$
\left|D^{\alpha} b(\xi)\right| \leq C_{0}|\xi|^{-(n+1) / 2-\varepsilon-|\alpha|}, \quad|\alpha| \leq n+3 .
$$

Then one has the inequality

$$
\left|\int b(\xi) e^{i[\langle x, \xi\rangle+\Phi(\xi)]} d \xi\right| \leq C(1+|x|)^{-n-1} .
$$

Furthermore the constant $C$ depends only on $\varepsilon, C_{0}, C_{0}$ and the upper bounds of the derivatives of order $\leq n+3$ of $\Phi$ on $S^{n-1}$.

PROOF OF LEMMA 3.5. Let $I(x)$ be the left-hand side of (3.12). Then if $d \sigma$ is the induced Lebesgue measure on $S$, a change of variables implies that

$$
|I(x)| \cong\left|\int_{1}^{\infty} r^{-(n+1) / 2-\varepsilon}\left\{\int_{S} \psi(r, \eta) e^{2 \pi i r\langle x, \eta\rangle} d \sigma(\eta)\right\} r^{n-1} d r\right|,
$$

where $\psi(r, \cdot)$ is in a bounded set of $C^{\infty}(S)$. Therefore since $S$ is convex, stationary phase implies that (see e.g. [4, Vol. I, p. 228])

$$
|I(x)| \leq C \int_{1}^{\infty} r^{-(n+1) / 2-\varepsilon}|x r|^{-(n-1) / 2} r^{n-1} d r=C^{\prime}|x|^{-(n-1) / 2} .
$$

Also, if $|x|$ is small enough then $\left|\nabla_{\xi}\{\Phi(\xi)+\langle x, \xi\rangle\}\right| \leq c$ and so an integration by parts argument would give $|I(x)| \leq C$ for such $x$. Consequently, we have now shown that $|I(x)| \leq C$ for some $C<\infty$.

To obtain the estimate for large $x$, we notice that if $x$ is large enough one has $\left|\nabla_{\xi}\{\Phi(\xi)+\langle x, \xi\rangle\}\right| \leq c|x|$ and so another integration by parts argument will show that $|I(x)| \leq C|x|^{-n-1}$ for these $x$.

This completes the proof of Lemma 3.5, and consequently finishes the proof of Theorem 3.1.

4. The Cauchy problem for strictly hyperbolic operators of order $m \geq 3$. In this section we stick to the notation of the previous one and our main result is the following. 
THEOREM 4.1. Let $\gamma(s, x)=\left(\gamma_{0}(s, x), \ldots, \gamma_{n}(s, x)\right)$ be a smooth family of nontangential curves in $\mathbf{R}^{n+1}, n \geq 1$. Also let $L$ be a strictly hyperbolic operator of order $m \geq 3$ as above which satisfies (3.2). Then if $f \in L_{\text {loc }}^{2}\left(\mathbf{R}^{n}\right)$, the (weak) solution $u(t, x)$ of the Cauchy problem (3.1) satisfies

$$
u(\gamma(s, x)) /\left(\gamma_{0}(s, x)\right)^{m-1} \rightarrow c_{\gamma}(x) f(x) \quad \text { as } s \rightarrow 0_{+}
$$

almost everywhere; the smooth function $c_{\gamma}$ is given by

$$
c_{\gamma}(x)=\left\{(m-1) !\left[\left(\partial \gamma_{0} / \partial s\right)(0, x)\right]^{m-1}\right\}^{-1} .
$$

In view of Lemma 3.2, Theorem 4.1 follows from Theorem 1.1 by obvious modifications of the $L^{2}$ arguments of the previous section which we leave to the reader.

REMARKS. (i) The analogue of Theorem 4.1 for operators $L$ of order $m=1$ is false. In fact, consider the case where $n=1$ and the strictly hyperbolic operator $L$ is given by $L=\partial / \partial t-\partial / \partial x$. Then the Cauchy problem $L u=0, u(0, x)=f(x)$ is solved by putting $u(t, x)=f(x+t)$ and so clearly (4.1) cannot hold for $L_{\text {loc }}^{2}$.

(ii) If one uses an interpolation argument similar to that of the previous section one can prove that for $m \geq 3$ there is an $\varepsilon=\varepsilon_{m, n}>0$ so that whenever $L$ is as in Theorem 4.1, it follows that (4.1) holds for $f \in L_{\text {loc }}^{p}\left(\mathbf{R}^{n}\right), p>2-\varepsilon$. This method, however, does not obtain sharp results and it seems that finding an analogue of Theorem 1.1 for $1<p<2$ would be a better approach.

(iii) If instead of (3.1) one considers the modified initial value problems

$$
\left\{\begin{array}{l}
L u=0 \\
\left(\partial^{j} u / \partial t^{j}\right) u(0, x)=0, \quad 0 \leq j<l<m-1, \\
\left(\partial^{l} / \partial t^{l}\right) u(0, x)=f(x)
\end{array}\right.
$$

(note that the derivatives of order $l<j \leq m-1$ have not been specified) then if $f \in L_{\text {loc }}^{2}\left(\mathbf{R}^{n}\right)$ there is a (weak) solution $u(t, x)$ of $(3.1 . l)$ for which

$$
u(t, x) / t^{l} \rightarrow f(x) / l !
$$

almost everywhere (and also the analogue of (4.1) holds for nontangential curves). In fact, there is a solution $u(t, x)$ for which the analogue of Lemma 3.2 holds except that for this solution the amplitudes $a_{k}(t ; x, \xi)$ will only satisfy $(1.2)$ with $\delta=l-1$ (see e.g., [12, pp. 308-313]).

ACKNOWLEDGMENT. This paper grew out of discussions with E. M. Stein on related results. The author wishes to thank him for his advice.

\section{REFERENCES}

1. R. M. Beals, $L^{p}$ boundedness of Fourier integral operators, Mem. Amer. Math. Soc., No. 264, Amer. Math. Soc., Providence, R.I., 1982.

2. R. Courant and D. Hilbert, Methods of mathematical physics, Vol. II, Interscience, New York, 1982.

3. A. Greenleaf, Principal curvature and harmonic analysis, Indiana Math. J. 30 (1982), 519-537.

4. L. Hörmander, The analysis of linear partial differential operators, Vols. I-IV, Springer-Verlag, New York and Berlin, 1983, 1985.

5. P. D. Lax, Asymptotic solution of oscillatory initial value problems, Duke Math. J. 24 (1957), $627-646$.

6. A. Ruiz, On a.e. convergence of solutions of hyperbolic equations to $L^{p}$-initial data, Trans. Amer. Math. Soc. 287 (1985), $167-188$. 
7. C. D. Sogge and E. M. Stein, Averages of functions over hypersurfaces in $\mathbf{R}^{n}$, Invent. Math. $82(1985), 543-556$.

8. E. M. Stein, Maximal functions: spherical means, Proc. Nat. Acad. Sci. U.S.A. 73 (1976), 2174-2175.

9. E. M. Stein and S. Wainger, Problems in harmonic analysis related to curvature, Bull. Amer. Math. Soc. 84 (1978), 1239-1295.

10. E. M. Stein and G. Weiss, Introduction to Fourier analysis on Euclidean spaces, Princeton Univ. Press, Princeton, N.J., 1971.

11. M. Taylor, Pseudodifferential operators, Princeton Univ. Press, Princeton, N.J., 1981.

12. F. Treves, Introduction to pseudodifferential and Fourier integral operators, Vol. II, Plenum Press, New York and London, 1982.

Department of Mathematics, University of Chicago, Chicago, Illinois 60637 